\title{
Adaptive Sliding Observers for Induction Motor Control
}

\author{
Somboon Sangwongwanich*, Shinji Doki*, Tsuyoshi Yonemoto*, \\ Takeshi FuruHAshi* and Shigeru OKuMA*
}

This paper presents design methods of adaptive sliding observers for induction motor control, which include both the rotor speed and the rotor resistance identification together with the rotor flux estimation. The proposed adaptive observers have the advantage of their global stability over the conventional identification algorithms which are developed on restrictive assumptions. The primary reason for the simplicity of the analysis is the separation of the identifier from the controller, which is not realizable in the conventional algorithms.

Key Words: adaptive sliding observer, induction motor, sensor-less, identification

\section{Introduction}

To attain a high performance control of induction motors, it is necessary to know the exact value of the rotor flux. As parameter deviations of induction motors cannot be neglected, robust estimation of the rotor flux is indispensable. Recently, some researches on the robust estimation problem based on non-adaptive observers ${ }^{2), 6)}$ were reported, and it was clarified that the robustness of the non-adaptive observers has a limit which depends on the magnitude of parameter deviations. Moreover, in the indirect field-oriented controller, the rotor resistance, which is used in the slip frequency calculation, changes with temperatures. Further improvement on the robustness of both the observer and the indirect field-oriented controller can only be achieved by adaptive approaches.

Another research topic concerning control of induction motors which cannot be overcome by the non-adaptive observers is the removal of speed sensors to improve the mechanical robustness of the induction motor system. For this kind of system, the rotor flux must be estimated without the knowledge of the rotor speed, and the true rotor speed must also be simultaneously identified.

To cope with the problems just mentioned, several researches on the parameter (including the

\footnotetext{
* Faculty of Engineering, Nagoya University,

Furo-cho, Chikusa-ku, Nagoya, Japan

(Received August 20, 1990)

(Revised February 12, 1991)
}

rotor speed) identification of induction motors were carried out, and various kinds of identification schemes were proposed ${ }^{8) \sim 11)}$. Most of the adaptive schemes adjust the estimated parameters or the rotor speed using the error between the commanded torque current (slip frequency) and the real torque current (slip frequency). However, the convergence of the parameter identification and the stability of the identifier were not analyzed completely because of the following restrictive assumptions made in the analysis:

- The convergence of the estimated parameter to the true parameter was analyzed based on the assumption that the rotor flux was calculated correctly using an integrator.

- The stability of the identifier was analyzed by linearization around an operating point or at a steady state.

The aim of this paper is to develop adaptive observers which guarantee the global stability and the convergence of the estimated parameter taking into account the error in the rotor flux estimation. Firstly, corresponding to the aforementioned two estimation problems, we propose the following two adaptive sliding observers :

[1] An adaptive rotor flux observer which possesses the rotor speed identifier.

[2] An adaptive rotor flux observer which possesses the rotor resistance identifier.

The advantages of the proposed adaptive sliding observers are as follows:

- The identifier and the controller are separated (Fig. 1).

- The global stability is guaranteed even when the error in the rotor flux estimation exists.

- The rotor flux is estimated by stable observers, therefore the proposed adaptive observers confront no problem in the low speed region where the conventional ones suffer a lot from the drift of the integrator.

Secondly, we proposed a new construction of the adaptive controller. Although the indirect type of the field-oriented control has so far found favor in the conventional adaptive controllers, it should be noted that, in the speed-sensor-less drives, the error in the identification of either the rotor speed 


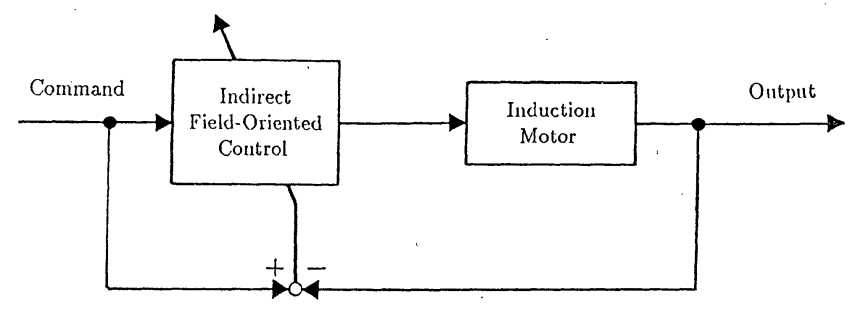

(a) Conventional adaptive controller

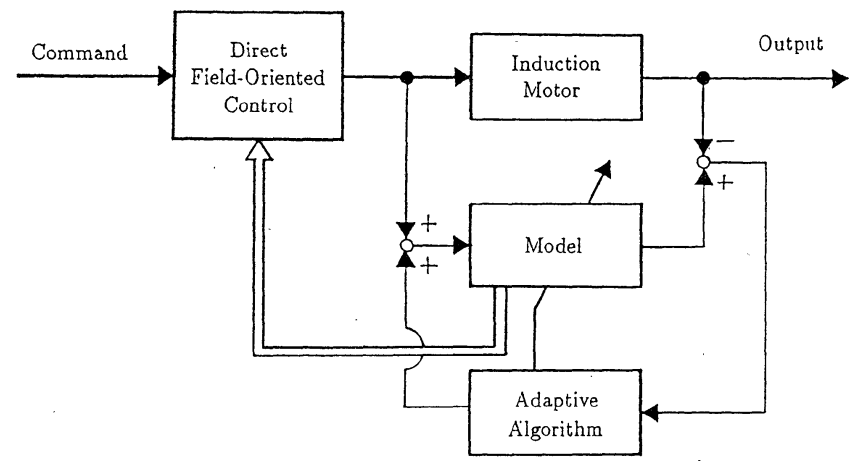

(b) Proposed adaptive controller

Fig. 1 Configuration of adaptive controller for induction motor

or the rotor resistance will degrade the control characteristics of the system, because the error is accumulated by the integrator used in computing the phase of the rotor flux. In this paper, the direct field-oriented controller is adopted considering its robustness against the rotor resistance and rotor speed estimation error.

\section{Sliding Observers of Induction Motor}

Sliding observers have long been known for its capability of disturbance identification and low dimension property ${ }^{3), 5)}$. Although the adaptive sliding observer of a simple linear system whose states are all measurable was first proposed by Utkin ${ }^{3)}$, the extension of the theory to a general linear system has not received much attention so far. Concerning the induction motor drives, the authors have previously proposed an sliding observer for robust estimation of the rotor flux of induction motors ${ }^{6)}$, and have also found that the identification capability is useful in designing an adaptive observer. In this section, the construction of a sliding observer for an induction motor and some of its characteristics are reviewed.

\subsection{Construction of Sliding Observers}

The state equations of the induction motor in rectangular $(d-q)$ coordinates fixed to the stator are given by

$$
\frac{d}{d t}\left[\begin{array}{l}
i_{s} \\
\lambda_{r}
\end{array}\right]=\left[\begin{array}{ll}
A_{11} & A_{12} \\
A_{21} & A_{22}
\end{array}\right]\left[\begin{array}{c}
i_{s} \\
\lambda_{r}
\end{array}\right]+\left[\begin{array}{c}
B_{1} \\
0
\end{array}\right] v_{s}
$$

where

$$
\begin{aligned}
& A_{11}=-\left(R_{s}+R_{r} M^{2} / L_{r}^{2}\right) /\left(\sigma L_{s}\right) \cdot I \\
& A_{12}=\left(R_{r} M / \sigma L_{s} L_{r}^{2}\right) \cdot I-p \omega_{m} M /\left(\sigma L_{s} L_{r}\right) \cdot J \\
& A_{21}=\left(M R_{r} / L_{r}\right) \cdot I \\
& A_{22}=-\left(R_{r} / L_{r}\right) \cdot I+p \omega_{m} \cdot J=-\varepsilon A_{12} \\
& B_{1}=\left(1 / \sigma L_{s}\right) \cdot I, \quad \sigma=1-M^{2} /\left(L_{s} L_{r}\right) \\
& \varepsilon=\sigma L_{s} L_{r} / M \\
& I=\left[\begin{array}{ll}
1 & 0 \\
0 & 1
\end{array}\right], \quad J=\left[\begin{array}{cc}
0 & -1 \\
1 & 0
\end{array}\right] .
\end{aligned}
$$

The state variables are the stator current $i_{s}$ and the rotor flux $\lambda_{r}$. The measurable output is $i_{s}$. The control input is the stator voltage $v_{s}$. Definitions of the parameters are given in Appendix. A sliding observer for the induction motor is constructed as

$$
\begin{aligned}
& \hat{i_{s}}=\hat{A}_{11} \hat{i}_{s}+\hat{A}_{12} \hat{\lambda}_{r}+B_{1} v_{s}+K_{1} \operatorname{sgn}\left(\hat{i}_{s}-i_{s}\right) \\
& \hat{\lambda}=\hat{A}_{21} \hat{i}_{s}+\hat{A}_{22} \hat{\lambda}_{r}-L K_{1} \operatorname{sgn}\left(\hat{i}_{s}-i_{s}\right)
\end{aligned}
$$

and the sliding hyperplane is

$$
\xi=\left[\begin{array}{ll}
\xi_{1} & \xi_{2}
\end{array}\right]^{T}=\hat{i}_{s}-i_{s}=0
$$

where ${ }^{\wedge}$ denotes the estimated value. The error equations of the sliding observer are obtained as

$$
\begin{aligned}
& \dot{e}_{1}=\hat{i}_{s}-\dot{i}_{s}=A_{11} e_{1}+A_{12} e_{2} \\
& +K_{1} \operatorname{sgn}\left(\hat{i}_{s}-i_{s}\right)+D_{1} w \psi \\
& \dot{e}_{2}=\dot{\lambda}_{r}-\dot{\lambda}_{r}=A_{21} e_{1}+A_{22} e_{2} \\
& -L K_{1} \operatorname{sgn}\left(\hat{i}_{s}-i_{s}\right)+D_{2} w \psi \\
& D w \psi=\left[\begin{array}{c}
D_{1} \\
D_{2}
\end{array}\right] w \phi=\left[\hat{A}_{i j}-A_{i j}\right]\left[\begin{array}{l}
\hat{i}_{s} \\
\hat{\lambda}_{r}
\end{array}\right] \\
& \text { : parameter mismatch term } \\
& \phi=\hat{\theta}-\theta: \text { parameter error } \\
& \theta: \text { parameter to be identified. }
\end{aligned}
$$


The switching gain $K_{1}$ is chosen large enough to guarantee the existence of the sliding mode.

\subsection{Characteristics of Sliding Observer on Sliding Hyperplane}

When the sliding mode occurs, the equivalent control method ${ }^{3)}$ can be applied, and

$$
e_{1}=\hat{i}_{s}-i_{s}=0, \quad \dot{e}_{1}=\dot{i}_{s}-\dot{i}_{s}=0
$$

are obtained. Hence, the characteristics of the sliding observer on the sliding hyperplane are given by

$$
\begin{aligned}
& \dot{e}_{2}=\left(A_{22}+L A_{12}\right) e_{2}+F w \phi \\
& F=\left[\begin{array}{ll}
L & I
\end{array}\right] D \text {. }
\end{aligned}
$$

By substituting Eq. (8) into Eq. (5), we obtain Eq. (11).

$$
z \equiv-K_{1} \operatorname{sgn}\left(\hat{i}_{s}-i_{s}\right)=A_{12} e_{2}+D_{1} w \phi
$$

Considering that $A_{22}=-\varepsilon A_{12}$ and that from Eq. (11) $A_{12} e_{2}=z-D_{1} w \phi$, Eq. (9) can be rewritten as

$$
\dot{e}_{2}=(L-\varepsilon I) z+\left(\varepsilon D_{1}+D_{2}\right) w \phi \text {. }
$$

Equations (11) and (12) are the key equations of the adaptive sliding observers which are discussed in the sequel.

In reality, the signal $z$ is obtained by low-pass filtering of the switching signal $-K_{1} \operatorname{sgn}\left(e_{1}\right)$. Since the cut-off frequency of the filter should be set high enough compared with the frequency band-width of the motor variables, the residual switching noise still remains mixing with the signal $z$. This fact must always be held in mind when considering the design of the adaptive observer, because the integration of the so obtained signal $z$ may result in a drift in parameter identification.

Physically, the signal $z$ is the error between the differential of $\hat{i}_{s}$ and $i_{s}$, hence it contains directly the information of the parameter mismatch. This is the salient feature of the sliding observer which enables the identification algorithm to be derived rather easily.

\section{Adaptive Identification Algorithm}

Based on the key Eqs. (11) and (12), two identification algorithms can be developed. The assumptions made here are

$$
\dot{\theta}=0, \quad \dot{A}_{12}=0 \text {. }
$$

In addition, for the rotor speed and the rotor resistance identification,

$$
\varepsilon D_{1}+D_{2}=0
$$

is satisfied.

\subsection{Identification Algorithm I}

Consider first a Lyapunov function $V$ defined by

$$
V=\frac{1}{2}\left(z^{T} z+\frac{1}{g} \phi^{2}\right)
$$

together with the following identification algorithm :

$$
\begin{aligned}
& \dot{\hat{\theta}}=\dot{\phi}=-g z^{T}\left[\Delta_{12}(L-\varepsilon I) z+D_{1} \dot{w}\right] \\
& \hat{\alpha}>\frac{a\left\|D_{1} w\right\| \cdot\left\|z^{T} \Delta_{12}(L-\varepsilon I) z+z^{T} D_{1} \dot{w}\right\|}{\|z\|}
\end{aligned}
$$

where

$$
\begin{aligned}
& \Delta_{12} \psi=A_{12}-\hat{A}_{12} \\
& -\hat{\alpha} I+\hat{\beta} J=\hat{A}_{22}+L \hat{A}_{12} \\
& g: \text { adaptive gain }>0 .
\end{aligned}
$$

$-\hat{\alpha} \pm j \hat{\beta}$ are the required closed-loop poles of the observer which are assigned using the estimation value of $A_{12}$, i. e. $\hat{A}_{12}$. With the identification algorithm stated,

$$
\begin{aligned}
& \dot{V} \leq-\gamma\|z\|^{2} \\
& \gamma=\hat{\alpha}-\frac{g\left\|D_{1} w\right\| \cdot\left\|z^{T} \Delta_{12}(L-\varepsilon I) z+z^{T} D_{1} \dot{w}\right\|}{\|z\|}
\end{aligned}
$$

can be obtained ${ }^{7)}$.

Although $z(t)$ is guaranteed to decrease to zero, the parameter error $\phi$ and the estimation error of the rotor flux $e_{2}$ need not approach zero. To assure that $\phi(t) \rightarrow 0$ and $e_{2}(t) \rightarrow 0$ as $t \rightarrow \infty$, additional identifiability conditions must be satisfied. The concrete conditions will be given later.

\subsection{Identification Algorithm II}

In the identification algorithm $I$, the real part of the closed-loop poles $\hat{\alpha}$ should be large to attain a fast convergence of the parameter error. However, this would result in a high sensitivity against disturbances not treated in the model, and hence has to be avoided ${ }^{6)}$. In the following, we derive another identification algorithm which relaxes the condition on $\hat{\alpha}$ and is more simple than the algorithm I.

Consider now a Lyapunov function $V$

$$
V=\frac{1}{2} \psi^{2}
$$

and the following identification algorithm (gradient algorithm)

$$
\dot{\phi}=-g\left(D_{1} w\right)^{T} z \quad(g>0)
$$

For simplicity, it is assumed that

$$
\left\|\left(D_{1} w\right)^{T} A_{12} e_{2}\right\| \leq\left\|\left(D_{1} w\right)^{T} w \phi\right\|
$$

is satisfied, then

$$
\begin{aligned}
\dot{V} & =\phi \dot{\psi} \\
& =-\phi \cdot g\left(D_{1} w\right)^{T}\left(A_{12} e_{2}+r v \phi\right) \leq 0
\end{aligned}
$$

is obtained, which guarantees that the parameter error approaches zero as $t \rightarrow \infty$. The restrictive condition (25) is, in fact, satisfied by almost all operating conditions. It should be noted that in the conventional algorithms, it is usually assumed that $e_{2}(0)=0$ and $\hat{\alpha}=0$. With such assumptions, the condition (25) is evidently satisfied, therefore the condition (25) is a more relaxed condition than that of the conventional ones. In fact, it can be verified that even when the condition (25) is violated, the proposed gradient algorithm II is locally stable. The global stability is further achieved if mild assumptions on the parameter mismatch term $D_{1} w \phi$ are satisfied ${ }^{7)}$. 


\section{Adaptive Sliding Observers for Speed-Sensor-Less Drives}

\subsection{Identification Algorithm I}

As stated previously, $\dot{\theta}=\dot{\omega}_{m}=0$ is assumed. The parameter mismatch term and the signal $z$ in this case are given by

$$
\begin{aligned}
& z=A_{12} e_{2}-\frac{p}{\varepsilon} J \hat{\lambda}_{r}\left(\hat{\omega}_{m}-\omega_{m}\right) \\
& w=-J \hat{\lambda}_{r} \quad \phi=\frac{p}{\varepsilon}\left(\hat{\omega}_{m}-\omega_{m}\right) \\
& \Delta_{12}=J, \quad D_{1}=I, \quad D_{2}=-\varepsilon I .
\end{aligned}
$$

Then, by substituting the above equations into Eqs. (16) and (17), the identification algorithm is obtained as

$$
\begin{aligned}
& \dot{\omega}_{m}=\frac{\varepsilon g}{p} \cdot z^{T} J\left[\hat{A}_{21} \hat{i}_{s}+\hat{A}_{22} \hat{\lambda}_{r}\right] \\
& \hat{\alpha}>g \cdot\left\|\hat{\lambda}_{r}\right\| \cdot\left\|\hat{A}_{21} \hat{i}_{s}+\hat{A}_{22} \hat{\lambda}_{r}\right\| .
\end{aligned}
$$

\section{2 Identification Algorithm II}

Considering Eqs. (27)-(29), the corresponding identification algorithm II is given by

$$
\hat{\omega}_{m}=\frac{g \varepsilon}{p} z^{T} J \hat{\lambda}_{r}
$$

It can be shown from Eq. (26) that, with the constant adaptive gain $g$, the convergence rate of the parameter error is proportional to $\|w\|^{2}=\left\|\hat{\lambda}_{r}\right\|^{2}$. Therefore, to attain uniformly a constant convergence rate $K_{I}$, the adaptive gain should be adjusted as :

$$
g=\frac{K_{I}}{\left\|\hat{\lambda}_{r}\right\|^{2}}
$$

If the controller keeps the rotor flux constant, Eqs. (32) and (33) will yield the same response. However, the distinction will be seen in the case that the rotor flux is varied. Furthermore, the identification algorithm can also be extended to include the proportional gain $K_{p}$ besides the integral gain $K_{I}$.

However, as will be shown later, the additional proportional gain $K_{p}$ only enhances the speed of response but does not contribute to the improvement of boundedness of the parameter error. On the contrary, as the residue of the switching noise is still mixing with the signal $z$, using directly the signal $z$ without passing through an integrator should be avoided.

Concerning the assignment of closed-loop poles $(-\hat{\alpha} \pm j \hat{\beta}), \hat{\alpha}$ should be set small so as not to result in dynamically large error in the estimated rotor flux which tends to violate the condition (25). Although both of the identification algorithms derived require no condition on $\hat{\beta}$, it was clarified that the estimation error caused by the parameter error with $D=[I-\varepsilon I]^{T}$, is minimized when the closedloop poles are placed on the real axis, i. e. $\hat{\beta}=0^{6)}$.
Therefore, hereafter $\hat{\beta}=0$ is adopted.

\subsection{Identifiability Condition of Rotor Speed}

The condition which guarantees that the estimated rotor speed converges to the true rotor speed even if $z(t)=0$ is considered in the following.

From Eqs. (11) and (12), if $z=0$, then

$$
\begin{aligned}
& e_{2}(t)=\mathrm{const} \\
& \hat{\lambda}_{r}(t)=\lambda_{r}(t)+\mathrm{const} \\
& \frac{p}{\varepsilon} J \hat{\lambda}_{r}(t)\left(\hat{\omega}_{m}-\omega_{m}\right)=A_{12} e_{2}(t)=\mathrm{const}
\end{aligned}
$$

are obtained. Therefore, unless $\lambda_{r}(t)$ is constant which means that $\hat{\lambda}_{r}(t)$ is also constant, Eq. (36) apparently contradicts Eq. (35) if $\hat{\omega}_{m} \neq \omega_{m}$. Consequently, the identifiability condition is given by

$$
\lambda_{r}(t) \neq \text { const } \text { and } \notin L_{2} \text {. }
$$

where $L_{2}$ denotes the class of signals with finite energy.

Furthermore, if $\Delta \omega_{m} \equiv \hat{\omega}_{m}-\omega_{m}=0, e_{2}(t)=0$ follows immediately from Eq. (36). Thus, the identifiability condition provides the convergence of both the rotor speed and the rotor flux to the true values. The identifiability condition just mentioned reveals the fact that the rotor speed is unidentifiable if the induction motor is driven by a dc input. This condition is also clear from the equivalent circuit of the induction motor.

\section{4 Boundedness of Estimation Error during Acceleration/Deceleration}

In reality, the induction motor is usually applied in variable speed drives, therefore the assumption (13) is rarely satisfied. It is self-evident that if the rotor speed is variable and no prior information about the mechanical system is available, the identification error is unavoidable however large the adaptive gain is. Nevertheless, it is expected that the identification error should be small if the convergence rate of the identification algorithm is high. In the following, we will show the boundedness of the identification error of the rotor speed when the rotor speed is time-varying, and clarify the relation between the identification error and the adaptive gains.

Firstly, as previously stated, the error in the estimation of the rotor flux is usually small with the poles of the sliding observer placed on the real axis and with a low enough $\hat{\alpha}$, therefore for simplicity $e_{2}(t) \approx 0$ is assumed. This assumption is satisfied if $e_{2}(0)=0$ and $\hat{\alpha}=0$ which are usually assumed in the conventional identification algorithms. Hence

$$
z=-\frac{p}{\varepsilon} J \hat{\lambda}_{r}\left(\hat{\omega}_{m}-\omega_{m}\right)
$$

is obtained. Considering also the proportional gain $K_{p}$, Eq. (32) can be rewritten as

$$
\dot{\omega}_{m}=-K_{I} \Delta \omega_{m}-K_{p} \Delta \dot{\omega}_{m}
$$




$$
\Delta \dot{\omega}_{m}=-\frac{K_{I}}{1+K_{p}} \Delta \omega_{m}-\frac{1}{1+K_{p}} \dot{\omega}_{m}
$$

Now let the following conditions be satisfied:

$$
\begin{aligned}
& \frac{K_{I}}{1+K_{p}}>0 \\
& \left|\dot{\omega}_{m}\right|<\mu(\text { const }) .
\end{aligned}
$$

The condition (41) is the necessary condition for the stability of the identifier which must always be satisfied. And the condition (42) is the natural condition on the bound of the acceleration/deceleration determined by mechanical load conditions. Then,

$$
\begin{aligned}
& V=\frac{1}{2} \Delta \omega_{m}^{2} \\
& \dot{V} \leq-\Gamma\left(\left|\Delta \omega_{m}\right|\right)
\end{aligned}
$$

where

$$
\begin{aligned}
& \Gamma\left(\left|\Delta \omega_{m}\right|\right)=\frac{K_{I}}{1+K_{p}}\left|\Delta \omega_{m}\right|\left[\left|\Delta \omega_{m}\right|-d_{\max }\right] \\
& d_{\max }=\frac{\mu}{\left|K_{I}\right|}
\end{aligned}
$$

are obtained, and the uniform ultimate boundedness of the identification error can be derived as :

$$
\begin{aligned}
& \left\|\Delta \omega_{m}\left(t_{0}\right)\right\| \leq d_{0} \\
& \rightarrow\left\|\Delta \omega_{m}(t)\right\| \leq d \text { for } d>d_{\max } t \geq t_{0}+t_{d} \\
& t_{d}= \begin{cases}\left(d_{0}^{2}-d^{2}\right) / 2 \Gamma(d) & \text { if } d_{0}>d ; \\
0 & \text { otherwise. }\end{cases}
\end{aligned}
$$

From the results just mentioned, it is clear that the bound of the identification error is determined only by the integral gain $K_{I}$ and is independent of the proportional gain $K_{p}$. The proportional gain $K_{p}$ contributes to the enhancement of the rate of convergence. If the initial identification error lies inside the ultimate bound, improving the rate of convergence is meaningless, since it only shortens the time required for the identification error to reach the ultimate bound, which is of no benefit. Both the bound of the identification error and the rate of convergence can be improved by increasing $K_{I}$, but a compromise with the effect of the switching noise should be considered.

\section{Adaptive Sliding Observers for Rotor Resistance $\mathbb{R}_{r}$ Estimation}

\subsection{Identification Algorithm I}

We shall develop the identification algorithm for the rotor resistance, following the same procedure as in the case of the rotor speed. Similarly, $\dot{R}_{r}=0$ is assumed in this case. The parameter mismatch term and the signal $z$ in this case are given by

$$
\begin{aligned}
& z=A_{12} e_{2}-\frac{M}{L_{r} \varepsilon}\left(\hat{i}_{s}-\hat{\lambda}_{r} / M\right)\left(\hat{R}_{r}-R_{r}\right) \\
& w=-\left(\hat{i}_{s}-\hat{\lambda}_{r} / M\right): \text { torque current } \\
& \psi=\frac{M}{L_{r} \varepsilon}\left(\hat{R}_{r}-R_{r}\right)
\end{aligned}
$$

$$
\Delta_{12}=-\frac{1}{M} I, \quad D_{1}=I, \quad D_{2}=-\varepsilon I .
$$

Then, substituting the above equations into Eqs. (16) and (17) yields the identification algorithm:

$$
\begin{gathered}
\dot{\hat{R}}_{r}=-\frac{g \varepsilon L_{r}}{M^{2}} \cdot z^{T}\left[(M+\varepsilon) z+\left(\hat{A}_{21}-M \hat{A}_{11}\right) i_{s}^{*}\right. \\
\left.\quad+\left(\hat{A}_{22}-M \hat{A}_{12}\right) \hat{\lambda}_{r}-M B_{1} v_{s}\right] \\
\hat{\alpha}>\frac{g}{M}\left\|i_{s}^{*}-\hat{\lambda}_{r} / M\right\| \cdot \|(M+\varepsilon) z+\left(\hat{A}_{21}-M \hat{A}_{11}\right) i_{s}^{*} \\
\quad+\left(\hat{A}_{22}-M \hat{A}_{12}\right) \hat{\lambda}_{r}-M B_{1} v_{s} \| .
\end{gathered}
$$

It should be noted that the commanded current $i_{s}^{*}$ is used instead of the estimated one, $\hat{i}_{s}$, in Eqs. (52) and (53). The commanded current is identical to the fundamental component of the estimated current. However, due to the sliding mode the estimated current contains also the switching noises while the commanded current does not. Therefore, if the estimated current is used in Eq. (52), the product term between $\hat{i}_{s}$ and $z$ may result in an unexpected low frequency drift because both signals contain switching noises.

\section{2 Identification Algorithm II}

Considering Eqs. (48)-(51), the corresponding identification algorithm II is given by

$$
\hat{\hat{R}}_{r}=\frac{g \varepsilon L_{r}}{M^{2}} z^{T}\left(i_{s}^{*}-\hat{\lambda}_{r} / M\right) .
$$

Similarly, with the constant adaptive gain $g$, the convergence rate of the parameter error is proportional to $\|w\|^{2}=\left\|i_{s}^{*}-\hat{\lambda}_{r} / M\right\|^{2}=(\text { torque current })^{2}$. In contrast to the rotor speed identification, the torque current usually varies with time during acceleration/deceleration, therefore the convergence rate also varies with the torque current, which is undesirable. The identification algorithm can be redefined so as to attain uniformly a constant convergence rate as in the rotor speed case.

\subsection{Identifiability Condition of Rotor Resistance}

In the following, we will consider the condition on the identifiability of the rotor resistance when the signal $z$ becomes 0 . From Eq. (11), if $z=0$, then

$$
\begin{aligned}
& e_{2}(t)=\text { const } \\
& \hat{\lambda}_{r}(t)=\lambda_{r}(t)+\text { const }
\end{aligned}
$$

Also, by substituting $z=0$ into Eq. (48) and noting that $e_{2}(t)$ is constant, then the following equation is obtained.

$$
\begin{aligned}
& \frac{M}{\varepsilon L_{r}}\left(\hat{i}_{s}-\hat{\lambda}_{r} / M\right)(t)\left(\hat{R}_{r}-R_{r}\right) \\
& \quad=A_{12} e_{2}(t)=\mathrm{const}
\end{aligned}
$$

are obtained. The identifiability condition is given by

$$
\left[\hat{i}_{s}-\hat{\lambda}_{r} / M\right](t) \neq \text { const and } \notin L_{2}
$$

The above condition excludes also the case where the torque current is zero, which is self-evident, since no information of the rotor resistance is avail- 
able. It should be noted that although the rotor resistance is not identifiable with no torque current, the rotor flux still converges to the true value as the parameter mismatch term also vanishes for such a condition. The identifiability condition just mentioned reveals that the rotor resistance is unidentifiable when

- the induction motor operates at a constant speed with no loads (a well-known fact), and

- when the motor is driven by a dc input. This condition is satisfied, for example, when the motor is operated at standstill.

\section{Simulation and Discussions}

In this section, simulation is carried out to verify the feasibility of the proposed identification algorithm. As will be shown later, the identification algorithm II exhibits better characteristics than does the identification algorithm I, so the algorithm II will be mainly studied.

Firstly, the speed-sensor-less field-oriented control system is considered. As mentioned in the introduction, the indirect field-oriented control is apparently not suitable for speed-sensorless drives, since the identification error is inevitable during acceleration/deceleration, and the orientation of the current commands depends heavily on the information of the rotor speed. As the rotor flux is correctly estimated with the proposed adaptive observers, the direct field-oriented control, which is robust to the parameter errors in both the rotor speed and the rotor resistance, can then be constructed as shown in Fig. 2. The adaptive sliding observer is realized by the discretization of the state Eqs. (2) and (3) using simple Euler's approximation. The sampling time of the observer is set to be $25(\mu \mathrm{s})$ which is realizable with the digital signal processing (DSP) at present, and the sampling time of the field-oriented controller is 250 $(\mu \mathrm{s})$. The current controller is assumed to be a hysteresis comparator type with the hysteresis bandwidth of $0.5(\mathrm{~A})$. The dc voltage of the PWM inverter is $250(\mathrm{~V})$. The cut-off frequency of the low-pass filter, through which the signal $z$ is obtained, is set at $100(\mathrm{~Hz})$.

Simulation results of the system response at acceleration/deceleration with the identification algorithm I are shown in Fig. 3 wherein $\hat{\lambda}_{r}=\left(\hat{\lambda}_{d r}, \hat{\lambda}_{q_{r}}\right)$. As is seen, although the estimated rotor speed lags behind the true rotor speed, a good speed response is obtained. This is because the estimation error of the rotor flux is quite small. Though it is not shown here, when the indirect field-oriented controller is used instead, a fluctuation occurs in the response. The simulation results corresponding to the identification algorithm $I I$ are shown next in

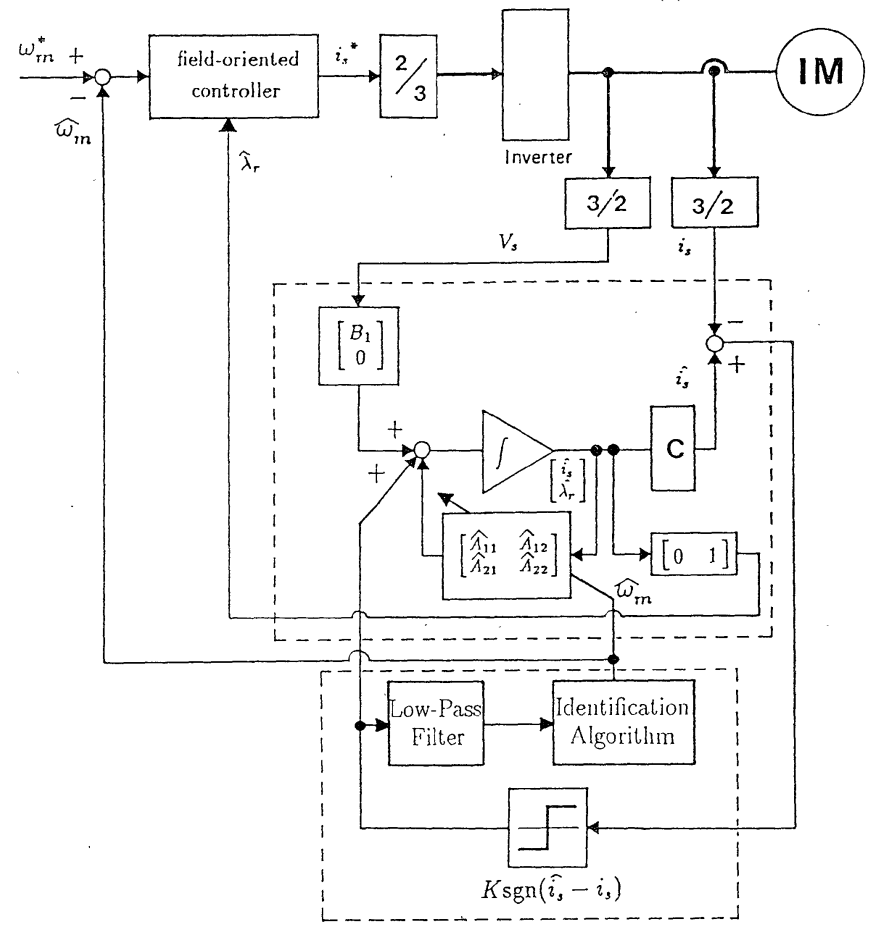

Fig. 2 Configuration of adaptive sliding observer for speedsensor-less drive
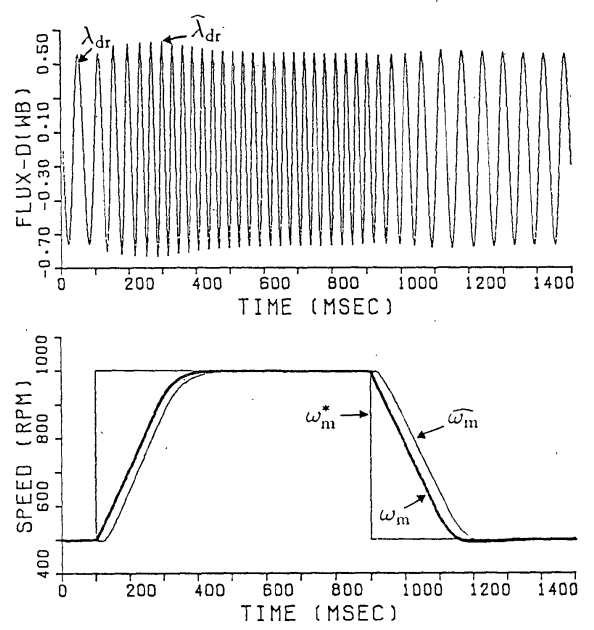

Fig. 3 Simulation results of speed-sensor-less drive with identification algorithm I 
Fig. 4. It is clearly seen that the rotor speed estimation exhibits almost no error, and a good speed response is obtained. The rotor flux is also correctly estimated owing to the small error in the rotor speed estimation. To indicate that the proposed identification algorithm II operates well also in the low speed region, simulation results in the
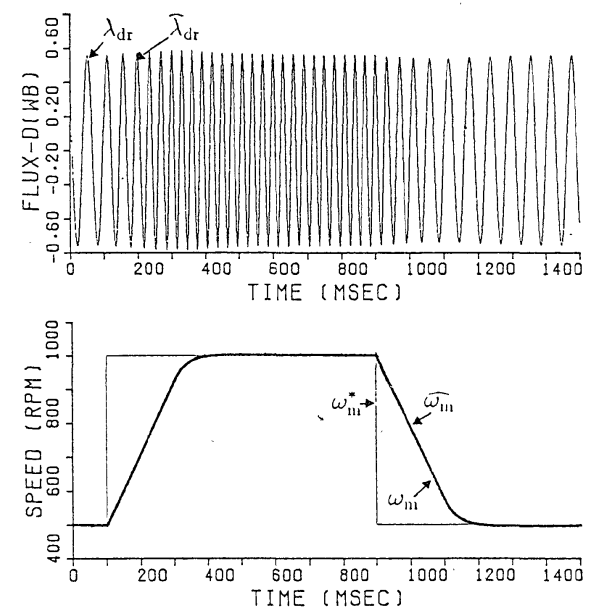

Fig. 4 Simulation results of speed-sensor-less drive with identification algorithm II (high speed region) case where the motor is started from stand-still to a commanded speed and then decelerated to standstill again are shown in Fig. 5. It is clear that the motor is controlled perfectly even at standstill.

Next, the direct field-oriented controller with a rotor resistance identification shown in Fig. 6 is considered. Fig. 7 shows the simulation results of
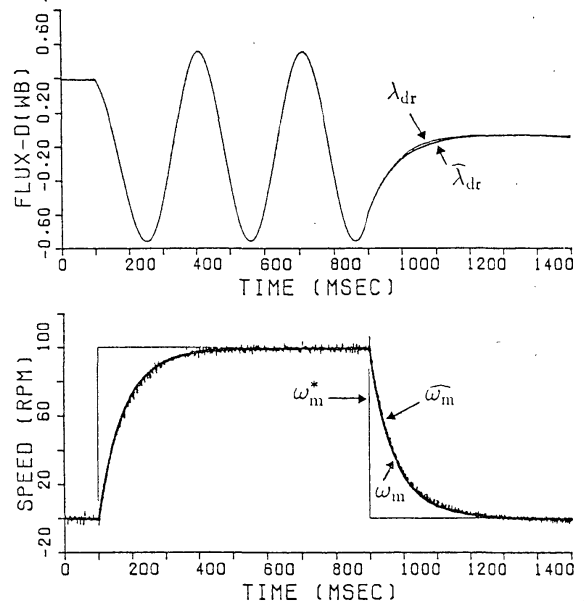

Fig. 5 Simulation results of speed-sensor-less drive with identification algorithm II (low speed region)

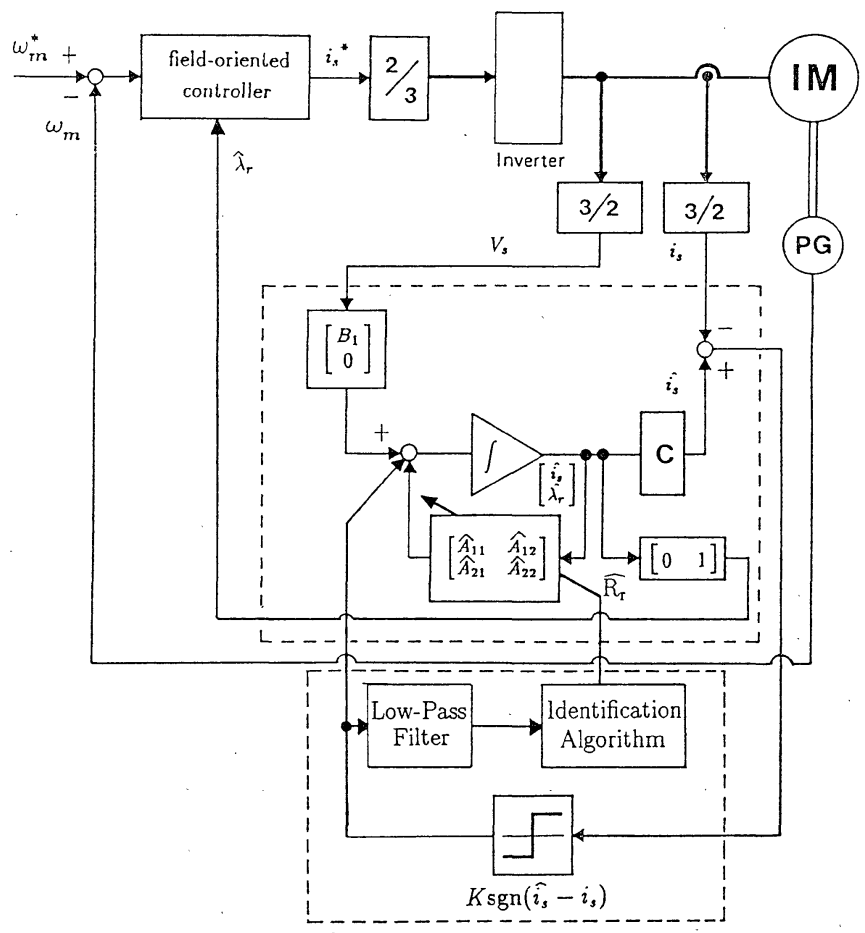

Fig. 6 Configuration of adaptive sliding observer for direct fieldoriented controller with rotor resistance identification 

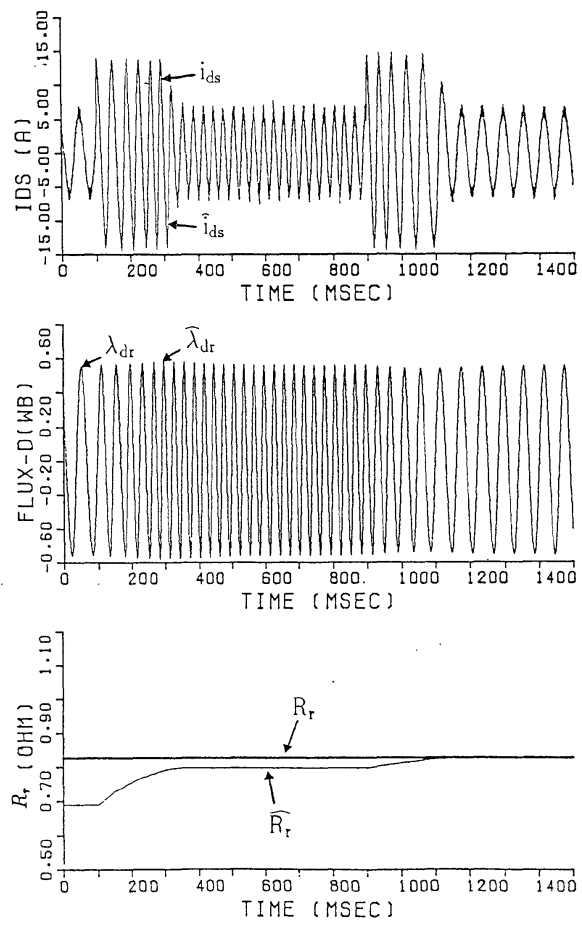

Fig. 7 Simulation results of direct field-oriented drive with rotor resistance identification

the identification algorithm II at acceleration/deceleration between $500(\mathrm{rpm})$ and $1,000(\mathrm{rpm})$. It is clear that the rotor resistance of the model is adjusted only during acceleration/deceleration where the torque current is non-zero, and that the parameter error converges to zero eventually.

\section{Conclusions}

The adaptive sliding observers for induction motor control have been presented in this paper to overcome the problem of instability of the rotor flux observer and the ambiguity of stability of the identifier which are the main disadvantages of the conventional adaptive schemes proposed until now. The results obtained can be summarized as follows :

1. Two stable identification algorithms are developed for the rotor speed and the rotor resistance identification.

2. The rotor flux is estimated by a stable observer which incorporates a stable rotor speed or rotor resistance identifier, and the problem of the drift of the integrator existing in the conventional schemes is solved.

3. For the rotor speed identification, the identification error during the acceleration/ deceleration is quantitatively evaluated, and the guidelines on the adaptive gain assignment is clarified.
4. It is pointed out that the field-oriented controller should be of the direct type which is robust to the errors of both the rotor speed and the rotor resistance.

5. With the proposed rotor speed identification algorithm, the speed-sensor-less drives of good speed response characteristics are attained for a very wide range of operations from high speed to standstill.

The authors would like to express their thanks to Prof. Y. Hosoe of Nagoya University for his valuable advice.

\section{References}

1) G. Verghese and S. R. Sander: Observer for Flux Estimation in Induction Machines, IEEE Trans. Industrial Electronics, IE-35-1, 85/94 (1988)

2) Y. Hori and T. Umeno: Implementation of Robust Flux Observer-Based Field Orientation (FOFO) Controller for Induction Machines, Conf. Record 1989 Ann. Meeting IEEE-IAS, 523/528 (1989)

3) V. Utkin: Principles of Identification in Sliding Modes, Dokl. Akad. Nauk SSSR, 257-3, 558/561 (1981)

4) J.J.E. Slotine, et al.: On Sliding Observers for Nonlinear Systems, ASME J. of Dynamic Systems, Measurement and Control, 109, 245/252 (1987)

5) J. Xu and H. Hashimoto: A VSS Observer for Nonlinear Systems, Proc. of SICE '88, ES1-4, 777/780 (1988)

6) S. Sangwongwanich, et al. : Design of Sliding Observers for Robust Estimation of Rotor Flux of Induction Motors, Int. Power Electronics Conference-Tokyo, 1235/1242 (1990)

7) S. Sangwongwanich, et al.: On Design of Adaptive Sliding Observers for Induction Motor Control, IEEE Int. Workshop on Advanced Motion Control, $8 / 17(1990)$

8) W. Sugimoto, et al.: Secondary Resistance Identification of an Induction Motor Applied Model Reference Adaptive System and Its Characteristics, IEEE Trans. Industry Applications, IA-23-2, 296/ 303 (1987)

9) H. Nakano and I. Takahashi: Sensor Less Field Oriented Control of an Induction Motor Using an Instantaneous Slip Frequency Estimation Method, Conf. Record of IEEE/PESC, 847/854 (1988)

10) T. Okuyama, et al.: Vector Control Scheme of Induction Motor without Speed and Voltage Sensors, IEEE Japan Trans., 107D-2, 191/198 (1987)

11) T. Ohtani, et al.: Approach of Vector-Controlled Induction Motor Drives without Speed Sensor, IEEE Japan Trans., 107D-2, 199/206 (1987)

12) S. Sastry and M. Bodson: Adaptive Control, Prentice Hall, New Jersey (1989)

\section{《Appendix》}

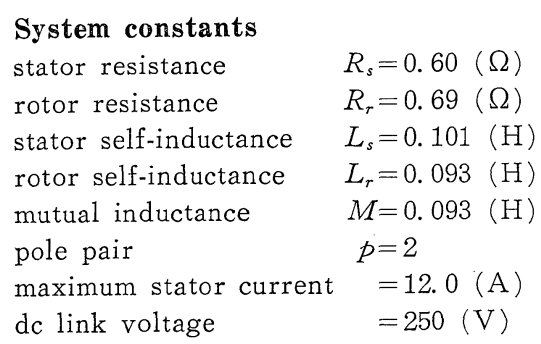

